Article

\title{
The Effect of Anti-COVID-19 Policies on the Evolution of the Disease: A Complex Network Analysis of the Successful Case of Greece
}

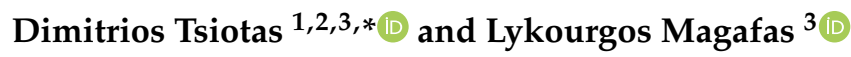 \\ 1 Department of Regional and Economic Development, Agricultural University of Athens, Greece, Nea Poli, \\ 33100 Amfissa, Greece \\ 2 Department of Planning and Regional Development, University of Thessaly, Pedion Areos, \\ 38334 Volos, Greece \\ 3 Laboratory of Complex Systems, Department of Physics, International Hellenic University, Kavala Campus, \\ 65404 St. Loukas, Greece; lmagafas@otenet.gr \\ * Correspondence: tsiotas@uth.gr; Tel.: +302-421-074-446; Fax: +302-421-074-493
}

Received: 22 April 2020; Accepted: 19 June 2020; Published: 22 June 2020

\begin{abstract}
Within the context of Greece promising a success story in the fight against the disease, this paper proposes a novel method for studying the evolution of the Greek COVID-19 infection curve in relation to the anti-COVID-19 policies applied to control the pandemic. Based on the ongoing spread of COVID-19 and the insufficient data for applying classic time-series approaches, the analysis builds on the visibility graph algorithm to study the Greek COVID-19 infection curve as a complex network. By using the modularity optimization algorithm, the generated visibility graph is divided into communities defining periods of different connectivity in the time-series body. These periods reveal a sequence of different typologies in the evolution of the disease, starting with a power pattern, where a second order polynomial (U-shaped) pattern intermediates, being followed by a couple of exponential patterns, and ending up with a current logarithmic pattern revealing that the evolution of the Greek COVID-19 infection curve tends towards saturation. In terms of Gaussian modeling, this successive compression of the COVID-19 infection curve into five parts implies that the pandemic in Greece is about to reach the second (decline) half of the bell-shaped distribution. The network analysis also illustrates stability of hubs and instability of medium and low-degree nodes, implying a low probability of meeting maximum (infection) values in the future and high uncertainty in the variability of other values below the average. The overall approach contributes to the scientific research by proposing a novel method for the structural decomposition of a time-series into periods, which allows removing from the series the disconnected past-data facilitating better forecasting, and provides insights of good policy and decision-making practices and management that may help other countries improve their performance in the war against COVID-19.
\end{abstract}

Keywords: coronavirus; pandemics; infectious disease; natural visibility algorithm; community detection; modularity optimization

\section{Introduction}

Starting in December 2019, in Wuhan city (China), the COVID-19 coronavirus disease is a new epidemic spreading virally across the world, causing cascading deaths and uncertainty for the future of the global and national economies [1-4]. Prime epidemiological studies on the COVID-19 infection and death curves [5-7] reveal the existence of strong scaling dynamics in the spreading of the disease, which appears to initiate under an exponential growth. On the other hand, other recent studies $[8,9]$ observe that this exponential growth is the first (incline) step of the Gaussian temporal spread describing 
epidemic phenomena. While the major concern of the research community and academia is obviously to find a cure for defeating this lethal pandemic [10,11], it is a consensus that (even when a laboratory formula will be soon available) the process of moving a cure from the laboratory to the production phase cannot be done at once. Therefore, beyond the global expectations that the day of a cure to this pandemic is forthcoming, governments worldwide are currently facing the challenge of managing their medical and human resources against the scaling (exponential-alike) trends of the disease-spreading [1,2,12]. The case of Italy was among the first countries dealing with COVID-19 outside the country of its origin, China. The uncontrolled spreading of the disease and the number of casualties [13] in Italy imply, on the one hand, the inertia of social life and structures to abrupt changes [14] and, on the other hand, the small-world [15-17] configuration of modern societies developing network structures. Although current scientific knowledge have led to the development of standardized protocols, best practices, and policies for controlling the pandemic $[18,19]$, countries worldwide show diverse patterns of conformance to these instructions, determining thus the evolution of the disease in their population in different ways. For instance, USA, UK, and Sweden, which appear to build up (at least initially) to the "herd immunity" strategy [20] are currently included in the top-ten countries in COVID-19 mortality rates [13], whereas others showing early emergence seem to succeed suspending their casualties.

Greece is an insightful case of good performance for its anti-COVID-19 policies, which appears to manage keeping the infected cases and deaths at relatively low levels [13,21], based on data up to the 54th day of the disease spreading. Specifically, Greece faced the first case of infection on February 26th, 2020, and just three days later it started applying several policies for controlling the disease. This timely alert and response have led Greece to be currently in the last places among European Countries in terms of infection and mortality rates of COVID-19 [13,21], setting the conditions to consider its case a success story in the war against the pandemic. Within the context of promising a success story, this paper studies the evolution of the COVID-19 infection curve in Greece in relation to the anti-COVID-19 policies applied by the state to control the disease, aiming to detect whether and how these policies affected the dynamics of the disease spreading. Given that the spread of COVID-19 in Greece corresponds to a period of less than two months, the classic time-series analysis approaches are inevitably subjected to high level of uncertainty for forecasting the future of the epidemic [22]. Additionally, prime quantitative approaches have already sufficed to reveal the exponential dynamics in the spreading of the disease [5-7]. Therefore, this paper advances current quantitative approaches by building on complex network analysis of time-series to transform the COVID-19 infection curve to a complex network. To do so, the visibility graph algorithm is applied [23], which is a non-parametric approach able to provide structural insights of the time-series and to transform it to a complex network that is a tensor of higher dimension in comparison to the series [22,24]. The association with the time-series visibility graph is detected for communities based on the modularity optimization algorithm [25], aiming to develop a partition of the time-series and therefore to interpret their significance in accordance with the anti-COVID-19 policies applied by the Greek state.

The overall approach first aims to provide insights of good policy and decision-making practices and management that may help other countries improve their performance against the pandemic. Secondly, it contributes to the scientific research by proposing a novel method for the structural decomposition of a time-series into periods that allow removing from the series the disconnected past data and thus it facilitates better forecasting. Both the proposed methodological framework and the case study of the success story of Greece (up to the 54th day of the disease spreading) can be proven effective in the war against COVID-19.

\section{Methodology and Data}

The methodological framework builds on complex network-based analysis of time-series to transform the time-series of the Greek COVID-19 infection curve into a complex network and then to examine the associated complex network instead of the time-series. The proposed methodological approach consists of four steps, as shown in Figure 1. 

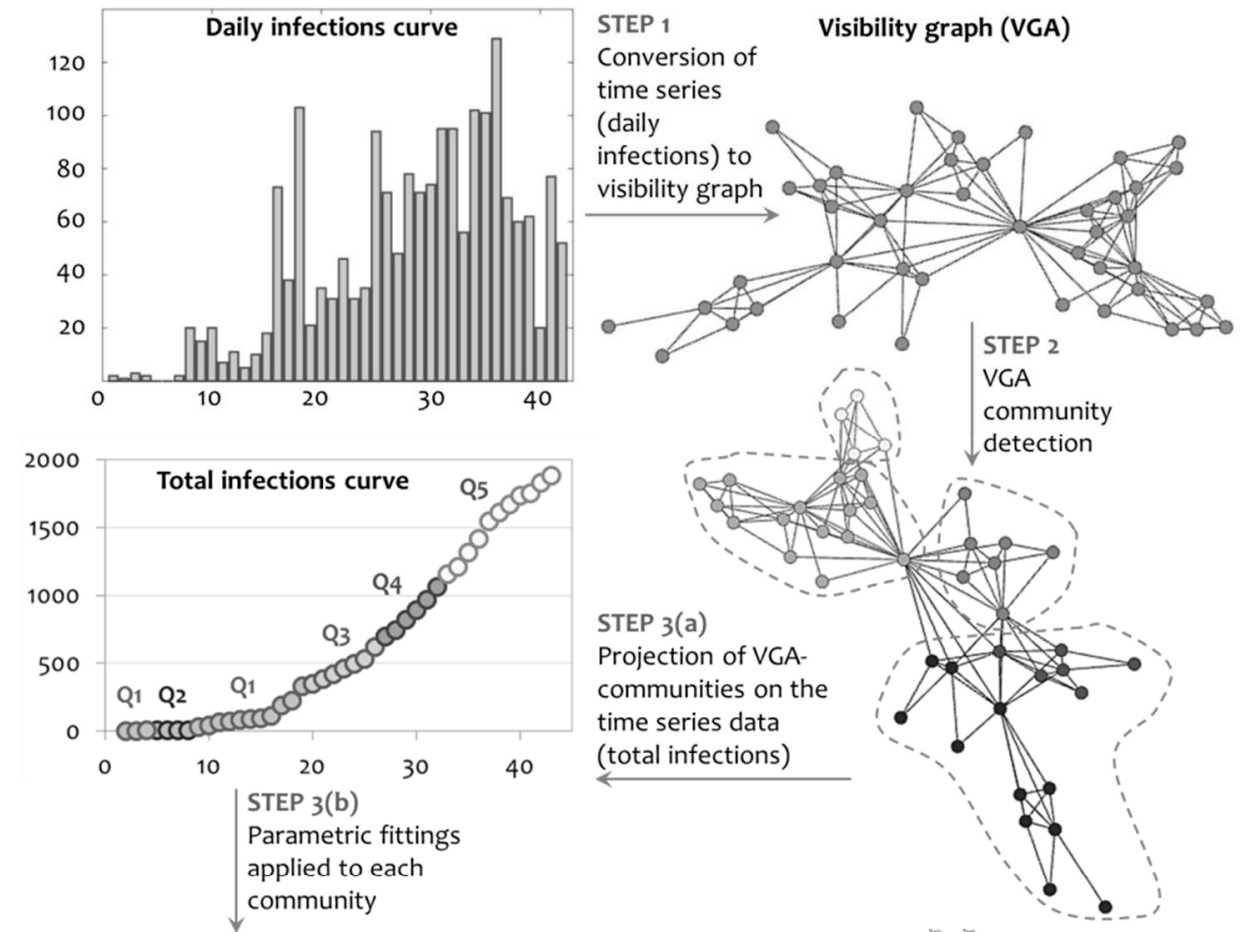

STEP 3(a)

Projection of VGAcommunities on the time series data (total infections)

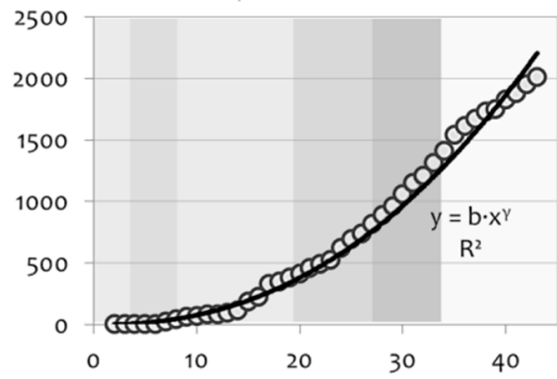

STEP 4

Policy evaluation within communities
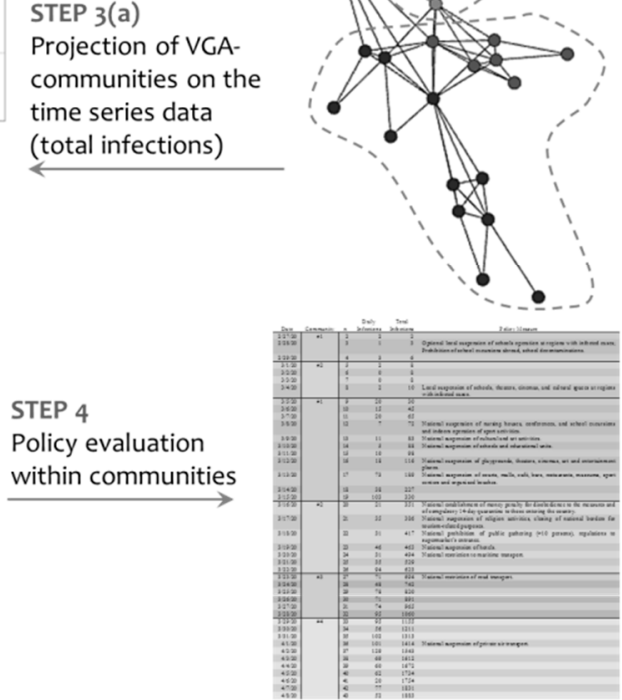

Figure 1. The methodological framework of the study.

At the first step (Figure 1), an associated visibility graph is generated based on data of the daily Greek COVID-19 infection curve. The available data were collected from daily announcements of the National Public Health Organization of Greece [26] and the Ministry of Health of Greece [27]. The database used in the analysis is not fully compatible with the GitHub repository (https://pomber. github.io/covid19/timeseries.json), which is obviously due to our own elaborations on these sources. The natural visibility algorithm (NVG) was proposed by the authors of [23] to transform a time-series into a graph (complex network) $G_{V}(V, E)$, where $V$ is the node-set and $E$ is the edge-set. The NVG builds on the intuition of considering the time-series as a path of successive mountains of different height (each representing the value of the time-series at the certain time). In this time-series-based landscape, an "observer" standing on a mountain can see (either forward or backwards) as far as no other mountain obstructs its visibility. In mathematical terms, each node $\left(t_{k}, x\left(t_{k}\right)\right)$ of the time-series corresponds to a graph node $n_{k} \equiv\left(t_{k}, x\left(t_{k}\right)\right) \in V$ (where the time-series order is preserved) and two nodes $n_{i}, n_{j} \in V$ are connected $\left(n_{i}, n_{j}\right) \in E$ when the following inequality (NVG connectivity criterion) is satisfied [23]:

$$
x\left(t_{k}\right)<x\left(t_{i}\right)+\left(x\left(t_{j}\right)-x\left(t_{i}\right)\right) \frac{t_{k}-t_{i}}{t_{j}-t_{i}}
$$

where $x\left(t_{i}\right)$ and $x\left(t_{j}\right)$ express the numerical values of the time-series nodes $n_{i} \equiv\left(t_{i}, x\left(t_{i}\right)\right)$ and $n_{j} \equiv\left(t_{j}\right.$, $\left.x\left(t_{j}\right)\right)$ and $t_{i}, t_{j}$ express their time-reference points. 


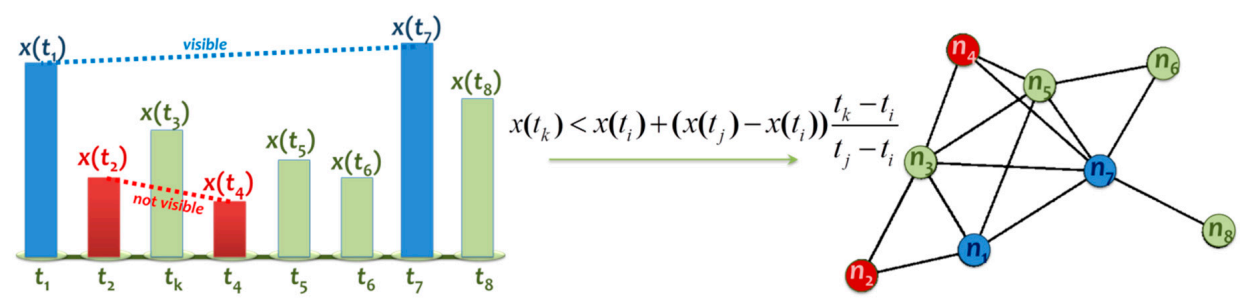

Figure 2. (left) Example visible and not visible pairs of nodes according to the natural visibility algorithm (NVG); (right) the visibility graph generated from the time-series shown at the left side.

In geometric terms, a visibility line can be drawn between two time-series' nodes $n_{i}, n_{j} \in V$ whether no other intermediating node $n_{k} \equiv\left(t_{k}, x\left(t_{k}\right)\right)$ obstructs their visibility [23,24], namely, no other intermediary node is so high as to intersect the visibility line created by this pair of nodes (Figure 2). Therefore, two time-series' nodes can enjoy a connection in the associated visibility graph if they are visible through a visibility line [23]. The visibility algorithm conceptualizes the time-series as a landscape and produces a visibility graph associated with this landscape [24]. The associated (to the time-series) visibility graph is a complex network where complex network analysis can be further applied [23,24].

In a network generated according to the visibility algorithm, each node is visible at least by its nearest (left and right) neighbors [24,28]. Studying the topology of the visibility graph instead of the time-series' structure benefits time-series analysis $[23,24,28]$ because it enlarges the space of the time-series embedding (a graph is a second-order tensor, whereas a time-series is a first-order tensor). In this broader space of embedding, complex network analysis can reveal latent information of the time-series structure that cannot be captured by classic time-series analysis. An effective complex network approach is the study of the degree distribution, which can provide useful insights about the hierarchical structure and the growth dynamics of the network topology $[15,17,29,30]$.

At the second step of the methodology (Figure 1), the associated visibility graph is first divided into communities, according to the modularity optimization algorithm of [25]. This algorithm produces several communities submitted to the criterion of maximizing the intra-community (i.e., within the communities) connectivity and therefore of minimizing the inter-community (between the communities) connectivity, as denoted by the mathematical expression Fortunato (2010):

$$
\text { maximize }\left[Q \propto\left(m_{\text {within communities }}-m_{\text {between communities }}\right)\right]
$$

where $Q$ is the modularity function [31] and $m$ the number of links.

The modularity optimization algorithm [25] is a greedy approach applied at two stages [31]. At the first, all graph nodes are assigned to different communities. Next, the nodes are sequentially swept and placed into collective communities if the assignment of a node into a neighbor community increases the gain in the weighted modularity function $\left(Q_{w}\right)$ of the initial graph. At the second stage, the collective communities are replaced by super-nodes and the procedure is repeated until the modularity function cannot increase any more [17,31]. In general, greedy algorithms are sensitive to local optima (e.g., the algorithm can be "trapped" within the region of a local optimum), and therefore they will not definitely lead to global optimum results [31]. Within this context, the resulting communities do not surely define the optimum partition of the network but a "very good" one, to the extent that nodes are densely connected within the communities and sparsely connected between them. Due to its greedy configuration, the algorithm is run several times and the final communities are resulted by the set theoretical intersection of all runs.

For a thorough review regarding modularity optimization and community detection in graphs, see the review article of [31].

At the third step of the methodology (Figure 1), the communities generated by the modularity optimization algorithm are projected to the time-series of the Greek COVID-19 total (cumulative) 
infections curve. This allows the time-series body to be divided into corresponding periods (communities), which represent strongly (or, more accurately, highly) connected sub-networks in the visibility graph. This allows fitting techniques to be applied to the time-series of each community and to examine their structure in terms of determination and forecasting. According to this approach [32], a parametric curve is fitted to the data of a variable $y=f(t)$ that bests describes its variability. The available types of fitting curves examined in the regression analysis are the linear (1st-order polynomial), quadratic (2nd-order polynomial), cubic (3rd-order polynomial), power, exponential, and logarithmic. All available types of fitting curves can be generally described by the general multivariate linear regression model defined by the mathematical expression [32]:

$$
\begin{aligned}
& \hat{y}=b_{1} f_{1}(x)+b_{2} f_{2}(x)+\ldots+b_{n} f_{n}(x)+c= \\
& =\sum b_{i} f_{i}(x)+c
\end{aligned}
$$

The $f(x)$ function can be either $\log$ arithmic $f(x)=(\log (x))^{m}$, or polynomial $f(x)=x^{m}$, or exponential $f(x)=(\exp \{x\})^{m}$, or any other. Within this context, the purpose of the curve fitting analysis is to estimate the $b_{i}$ parameters (in relation 3 ) that best fit to the observed data $\mathrm{y}$, so that to minimize the square differences $y_{i}-\hat{y}_{i}[32]$, according to the relation:

$$
\begin{aligned}
& \min \left\{e=\sum_{i=1}^{n}\left[y_{i}-\hat{y}_{i}\right]^{2}\right\}= \\
& =\min \left\{\sum_{i=1}^{n}\left[y_{i}-\left(\sum b_{i} f_{i}(x)+c\right)\right]^{2}\right\}
\end{aligned}
$$

The beta coefficients $\left(b_{i}\right)$ are estimated using the Least-Squares Linear Regression (LSLR) method [32], based on the assumption that the differences $e$ in Relation (3) follow the normal distribution $N\left(0, \sigma_{e}^{2}\right)$.

At the final step of the methodological framework (Figure 1), the Greek anti-COVID-19 policies included in each community are discussed. This step attempts to develop a policy profile for each community, in accordance with the anti-COVID-19 policies included in each period (community). Given that the time from exposition to the appearance of the symptoms of the disease ranges from 2 to 14 days, with a 5-day average [33-35], each policy applied within each period obviously affects the evolution of the infection curve with a respective time-lag. Therefore, the policies included in each period cannot be related to the shape of the infection curve of the corresponding period but probably to the next one (depending on the periods' lengths). However, the amount of policies included in each period can reveal in real-time the degree of the government's alert for the spreading dynamics of the disease and thus it can be considered as an indicator of the effectiveness of the public health management and policy in Greece. Therefore, this step of the methodology studies the amount of the Greek anti-COVID-19 policies within each period in the context that these policies describe the degree to which the state is alert to the spreading dynamics of COVID-19 in Greece and discusses these policies in accordance to the evolution of the curve observed at the next periods.

The dataset considered in the analysis begins with the starting day of the disease spreading in Greece (February 26th, 2020) and continues up to the 43rd day (April 4th, 2020). Each subsequent day, up to the 54th day of the disease spreading, is used to verify the forecasting in each community. In terms of testing, up to April 19th, Greece had conducted 53,290 tests and was ranked the 56th place worldwide and the 23rd place in Europe [33]. In the work of [33], the authors observed that testing in Greece was disproportional to the infection and the death curves of the disease, illustrating an aspect of ineffectiveness in the national health system. Therefore, the overall analysis is subject to the inevitable constraints of the country's testing policies. However, in the same work [33], the authors detect an almost perfect, positive, and significant (at the 0.01 level) correlation $(r=0.979)$ between the infection and deaths curves of the COVID-19 temporal spread in Greece. In the context in which the deaths curve is suggested to be a reliable indicator, because all deaths are absolutely recorded at all countries [33], the high and significant correlation $r=0.979$ captured by the authors of [33] between 
the Greek COVID-19 death and infection curves indicates that the analysis applied in this paper (based on the Greek COVID-19 infection curve) can be claimed to be based on trusted data.

Overall, the configuration of the proposed methodological framework advances time-series analysis because, firstly, it employs a double data consideration computed on daily (density or additive) and total (cumulative) infection data. This is because the associated visibility graph is generated on data of the daily COVID-19 infection curve (which are of higher variability), while parametric fitting is applied to the periods (communities) referring to the cumulative COVID-19 infection data (in which variability is smoother). This double consideration incorporates more information than (the one-variable approach) of analyzing a single time-series. Secondly, the proposed methodological framework builds on complex network analysis of time-series, which makes it possible to apply the modularity optimization algorithm to detect connected communities in the time series body. This approach incorporates information of network connectivity in the time-series structure that cannot be detected otherwise. Finally, the proposed methodological framework is indifferent to the case study of the Greek COVID-19 temporal spread; it can operate under insufficient data (here 43 cases) and can enjoy further applications.

\section{Results and Discussion}

\subsection{Network Analysis}

The degree distribution $p(k)$ of the associated with the Greek COVID-19 infection curve visibility graph $\left(G_{V}\right)$ is shown at Figure 3a. Among a set of available fittings (exponential, Fourier, Gaussian, linear, up to 4th-order polynomial, power, and logarithmic) that were tested [36] with the data, the normal (Gaussian) curve was found to have both the highest coefficient of determination [32] $\left(R^{2}=0.761\right)$ and the simplest expression (least number of estimated terms).

In comparison to a power-law curve, which is a particularly important fitting in network science for the detection of the scale-free property [17], and thus of hierarchical structures, the normal (Gaussian) curve with an estimated average $\hat{\mu}=\langle\hat{k}\rangle=4.491$ (number of connections per node) and an standard deviation $\hat{\sigma}=2.518$ better fits to the $p(k)$ data. The normal-shaped pattern of the degree distribution may have a double interpretation. On the one hand, it implies that the network is ruled by randomness because a normal curve asymptotically approximates the binomial distribution that theoretically describes the degree distribution of random networks [29]. On the other hand, the bell-shaped degree distribution implies that the majority of nodes have degrees close to the network's average, a fact that can also be related to the existence of spatial constraints. In particular, networks submitted to spatial constraints tend to develop neighborhood connections instead of distant ones, which are by default more costly and they are usually developed between the hubs [30,37]. Moreover, intense spatial constraints usually refer to planar network configurations [30], in which shortcuts or distant connections do not intersect the existing network links.
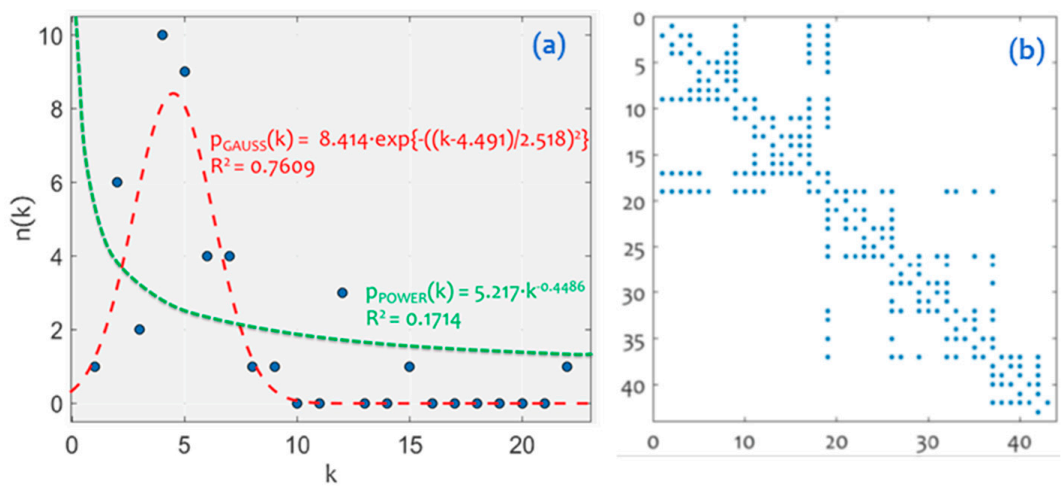

Figure 3. (a) The degree distribution $p(k)$ of the associated with the Greek COVID-19 infection curve visibility graph $G_{V}(n(k)$ denotes the frequency of degree $k) ;(\mathbf{b})$ the sparsity (spy) plot of $G_{V}$. 
In the literature [30,37], the most common network models with spatial constraints are the lattice or mesh graphs, which usually develop a regular tiling repeated throughout their structure. This regular structure causes the majority of nodes to have a degree near the average, leading to a bell-shaped degree distribution. Within this context, the degree distribution of the associated visibility graph implies that the network topology is more a mesh-like rather than a hierarchical one. To get more insights about the topology of the $G_{V}$, we examine the sparsity (spy) plot of this visibility graph (Figure $3 b$ ), which is a plot of the adjacency matrix displaying nonzero elements with dots [17]. The major concentration of the nonzero elements towards the main diagonal of the matrix implies that connections are mainly developed between neighbor nodes (the state of neighborhood is denoted in the adjacency by the successive labeling) and therefore that the visibility network is submitted to spatial constraints [17]. This observation verifies the previous finding based on the degree distribution consideration. However, the vertical spreading of dots in the spy plot may also indicate random trends [17], which is also in line with the previous observations.

According to the degree distribution and sparsity pattern analysis, the Greek COVID-19 visibility graph appears to be configured by random forces and spatial constraints. The emergence of randomness illustrates that future connections (which are created based on the daily infection values) may randomly attach to the network and therefore future maximum values of daily infection are possible. On the other hand, indications of spatial constraints (related to difficulty in developing distant connections) imply that future connections of the network are more dependent on their neighborhood values (i.e., the previous states of the time-series) and thus the phenomenon of the disease can resemble to a Markovian chain. However, regardless the insignificant level of determination $\left(R^{2}=0.171\right)$ of the power-law fitting (see [32,36]), a long-tail behavior of the degree distribution can be observed for degree values higher than $10(k>10)$, which expresses that the network structure of the $G_{V}$ is steadier for the hubs (i.e., nodes of high degree) than for medium- and low-degree nodes. This observation implies that the probability of meeting maximum (infection) values (and therefore the appearance of new hubs in the visibility network) in the future is considerably low, in contrast to the medium and low-degree nodes, where their future behavior is more uncertain.

\subsection{Community Detection}

\subsubsection{Structural Analysis}

In the second step, the infection curve visibility graph $\left(G_{V}\right)$ associated with the Greek COVID-19 is divided into communities, on which connectivity within each community is dense and between communities is sparse. The community discrimination of $G_{V}$ is shown in Figure 4, where labels of network nodes express the day since the first infection case. As can be observed, the community detection analysis of $G_{V}$ results to five communities (Figure 4). Running the community detection algorithm several times concludes with the same result, perhaps due to the relatively small amount of available time-series data (43 cases). These communities appear as a sequential ordering in the time-series body $\left(Q_{1}, Q_{2}, Q_{1}, Q_{3}, Q_{4}, Q_{5}\right)$, except the second one $\left(Q_{2}\right)$, which is injected into the first community (i.e., it is intermediate within the first community, according to the ordering $Q_{1}$, $\left.Q_{2}, Q_{1}, \ldots\right)$. Although this result is counterintuitive under a consecutive rationale, it supports the novelty of the proposed methodological approach, which is based on the connectivity assessment of the visibility graph generated on the additive (daily) time-series data. In particular, community $Q_{2}$ includes the elements (numbers of daily infections) $x\left(t_{5}\right)=2$ (day 5 ), $x\left(t_{6}\right)=0$ (day 6$), x\left(t_{7}\right)=0$ (day 7), and $x\left(t_{8}\right)=2$ (day 8 ), which appear in a symmetric arrangement $(2,0,0,2)$. This symmetry is captured by the modularity optimization of the visibility graph and resulted in the configuration of community $Q_{2}$. 


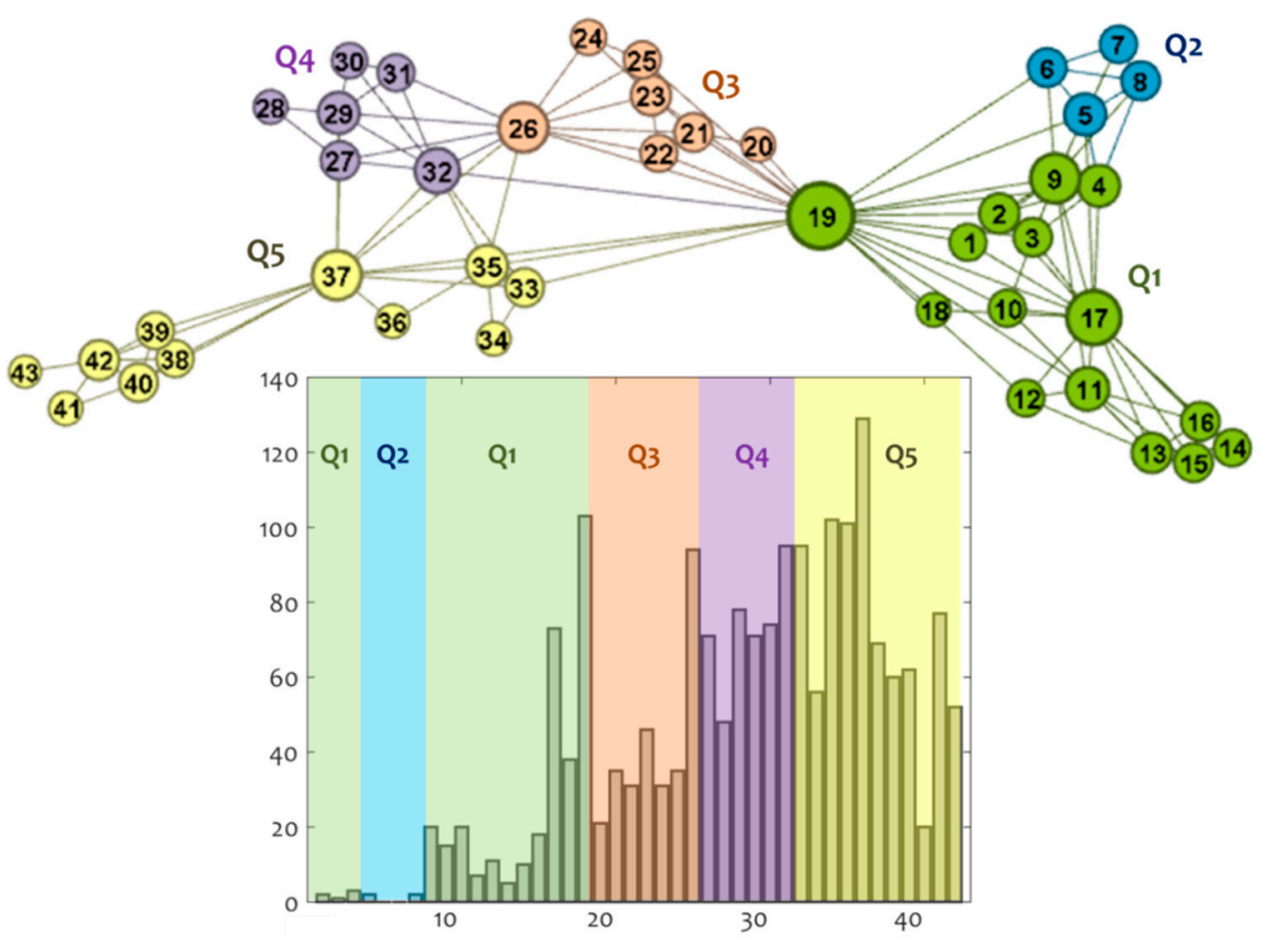

Figure 4. Division of the infection curve visibility graph $\left(G_{V}\right)$ associated with the Greek COVID-19 into five communities $\left(Q_{1}: Q_{5}\right)$ based on the modularity optimization algorithm of [25].

Next, Figure 5 is constructed by the projection of the modularity-communities onto the cumulative Greek COVID-19 infection curve. According to this figure, the evolution of the Greek COVID-19 infection curve is vertebral and consists of five time-periods corresponding to the modular communities shown in Figure 4. Parametric fittings were applied to all periods in accordance with the mathematical expressions shown in relations (3) and (4). The first $\left(Q_{1}\right)$ starts from the 1st day and ends up to the 19 th day (Figure $5 b$ ) of the infection spreading in Greece and is described by a scaling (power) pattern $\left(R^{2}=0.9799 \approx 0.98\right)$ with an exponent $\approx 2.21$. The second one $\left(Q_{2}\right)$ is injected into the first one and is extended to the time-interval $Q_{2}=\{5,6,7,8\}=\{5, \ldots, 8\} \subset \mathbb{N}$ (Figure 5 c), described by a (2nd-order) polynomial (U-shaped) pattern $\left(R^{2}=0.9333 \approx 0.93\right)$ with a positive coefficient $(\approx 0.5)$ of the highest order term. The third period $\left(Q_{3}\right)$ follows the first one, is extended to the time-interval $Q_{3}=\{20, \ldots, 26\}$ $\subset \mathbb{N}$ (Figure $5 \mathrm{~d}$ ), and is described by an exponential pattern $\left(R^{2}=0.9864 \approx 0.99\right)$ with an exponent $\approx 0.09$. Next, the fourth period $\left(Q_{4}\right)$ is extended to the time-interval $Q_{4}=\{27, \ldots, 32\} \subset \mathbb{N}$ (Figure 5 e) and it is also described by an exponential pattern $\left(R^{2}=0.9982 \approx 1\right)$, but with a smaller exponent than the previous period $(0.085<0.09)$. Finally, the fifth period $\left(Q_{5}\right)$ is extended to the time-interval $Q_{5}=\{33, \ldots, 43\} \subset \mathbb{N}$ (Figure $5 \mathrm{f}$ ) and is described by a logarithmic pattern $\left(R^{2}=0.9829 \approx 0.98\right)$.

Overall, according to the sequence of the modularity communities in the time-series body, the Greek COVID-19 infection curve initiated the first three days $\left(Q_{1}\right)$ with scaling (power) dynamics. Next, it showed an abrupt (U-shaped) tendency at the next four days $\left(Q_{2}\right)$, which was returned to the previous scaling (power) status $\left(Q_{1}\right)$, where it remained for the next 11 days $\{9, \ldots, 19\} \subset \mathbb{N}$. The next 13 days $\{20, \ldots, 32\} \subset \mathbb{N}$, the Greek COVID-19 infection curve showed an exponential growth, but in the mid-time of the period, the exponent decreased, and finally, in the last 11 days $(\{33, \ldots, 43\} \subset \mathbb{N})$ the infection curve showed a logarithmic trend, implying that the evolution of the infection curve was leading to saturation. 

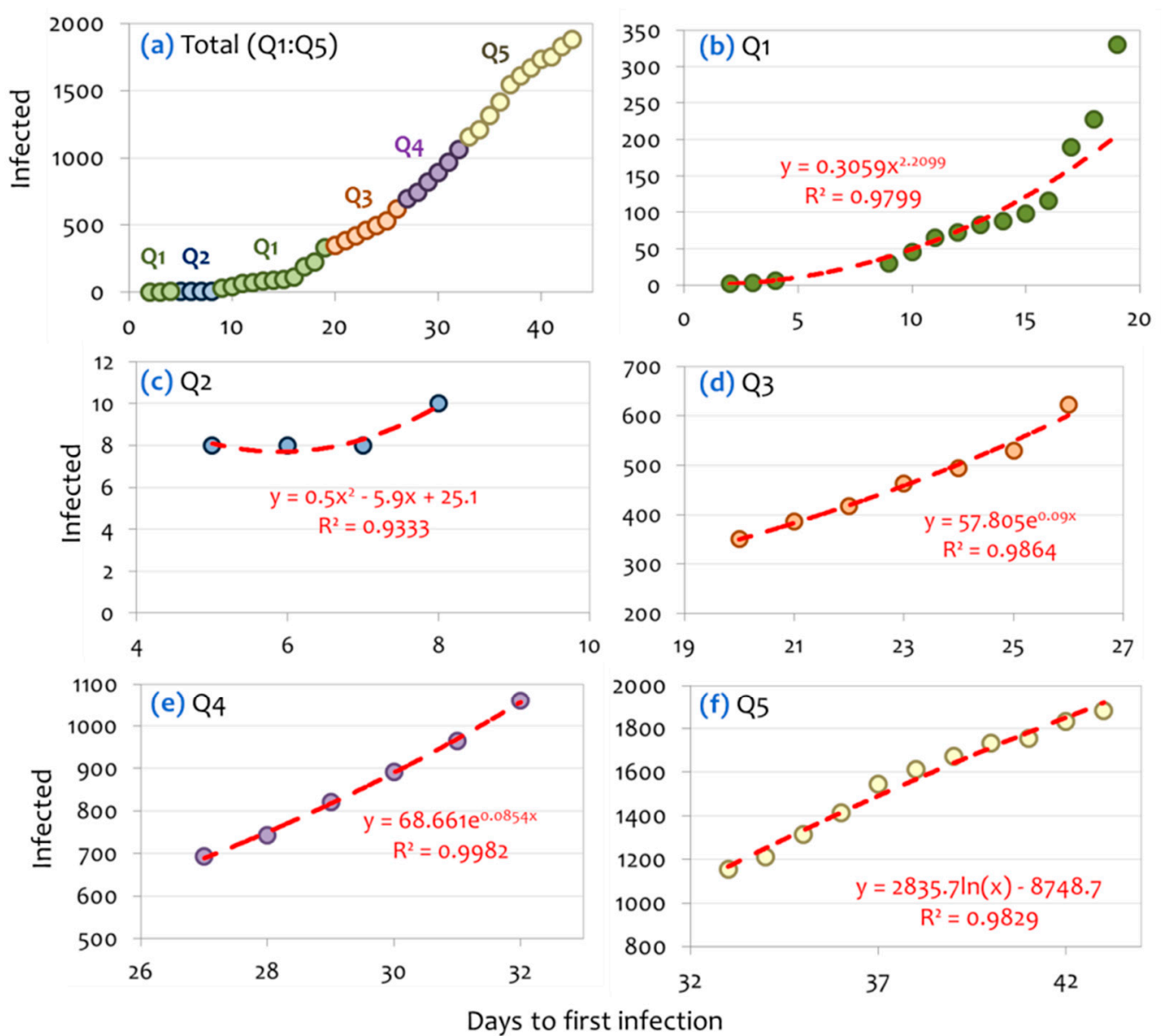

Figure 5. (a) The community structure of the Greek COVID-19 infection curve, where (b-f) show the best (of the highest $R^{2}$ ) fittings within each community.

\subsubsection{Forecasting}

In this paper, the analysis is based on the Greek COVID-19 infection curve, starting from the 1st day and ending at the 43rd day after the first infection (namely on the set $\{1, \ldots, 43\} \subset \mathbb{N}$ ). Cases after the 43rd day (up to the 54th day of COVID-19 temporal spread) are registered in Table 1 to test the estimations (as a verification set). Within this context, based on the fittings applied to each community $\left(Q_{1}: Q_{5}\right)$, future infections of the COVID-19 infection curve for the next 11 days were estimated and are shown in Table 1. As can be observed, the logarithmic curve of the last modularity community $\left(Q_{5}\right)$ provides the most accurate estimations in comparison with the five available communities and the total infection curve consisting of all communities $\left(Q_{1}: Q_{5}\right)$. Moreover, differences of most of communities seem to be of a certain magnitude $\left(\sim 600\right.$ for $Q_{1}, \sim 1200$ for $Q_{2}, \sim-1200$ for $Q_{3}, \sim-1100$ for $Q_{4}$, and $\sim-30$ for $Q_{5}$ ), which may be related to the effectiveness of the overall community detection approach. On the other hand, predictions based on the Gaussian model (GM) appear more accurate in the long term (i.e., in the total of the 11-day available future projections). This observation verifies relevant approaches, such as those of the authors of [8] and [9], which describe temporal spread in epidemic phenomena in terms of the Gaussian, bell-shaped, evolution. However, predictions of the last community's $\left(Q_{5}\right)$ model appear more accurate in the short-term (in particular for the first four days) and produce smaller mean square error (MSE). Overall, the joint (modularity-based and Gaussian) consideration implies that that the evolution of the pandemics in Greece is about to reach the peak-time and move to second (decline) half of the bell-shaped distribution. 
Table 1. Future cases of the COVID-19 cumulative infection curve (from 43rd to 54th day) based on the parametric fittings applied to each community $\left(Q_{1}: Q_{5}\right)$.

\begin{tabular}{|c|c|c|c|c|c|c|c|c|c|c|c|c|c|c|c|}
\hline \multirow{2}{*}{ Date } & \multirow{2}{*}{ Observed } & \multicolumn{14}{|c|}{ Estimations } \\
\hline & & $\mathrm{Q}_{1}$ & Diff & $\mathrm{Q}_{2}$ & Diff & $\mathrm{Q}_{3}$ & Diff & $\mathrm{Q}_{4}$ & Diff & $\mathrm{Q}_{5}$ & Diff & $\mathrm{Q}_{1: 5}$ (a) & Diff & $\mathrm{GM}^{(\mathrm{b})}$ & Diff \\
\hline $4 / 9 / 20$ & 1955 & 1311 & 644 & 734 & 1222 & 3032 & -1077 & 2942 & -987 & 1982 & $-27 *$ & 2194 & -239 & 2047 & -92 \\
\hline $4 / 10 / 20$ & 2011 & 1377 & 634 & 772 & 1239 & 3318 & -1307 & 3204 & -1193 & 2046 & -35 & 2325 & -314 & 2101 & -90 \\
\hline $4 / 11 / 20$ & 2081 & 1446 & 635 & 812 & 1269 & 3630 & -1549 & 3490 & -1409 & 2108 & -27 & 2461 & -380 & 2149 & -68 \\
\hline $4 / 12 / 20$ & 2114 & 1516 & 598 & 852 & 1262 & 3972 & -1858 & 3801 & -1687 & 2169 & -55 & 2601 & -487 & 2189 & -75 \\
\hline $4 / 13 / 20$ & 2145 & 1588 & 557 & 894 & 1251 & 4346 & -2201 & 4140 & -1995 & 2229 & -84 & 2747 & -602 & 2221 & -76 \\
\hline $4 / 14 / 20$ & 2170 & 1662 & 508 & 937 & 1234 & 4756 & -2586 & 4509 & -2339 & 2287 & -117 & 2897 & -727 & 2245 & -75 \\
\hline $4 / 15 / 20$ & 2192 & 1738 & 454 & 980 & 1212 & 5203 & -3011 & 4911 & -2719 & 2345 & -153 & 3052 & -860 & 2261 & -69 \\
\hline $4 / 16 / 20$ & 2207 & 1816 & 391 & 1025 & 1182 & 5693 & -3486 & 5349 & -3142 & 2401 & -194 & 3212 & -1005 & 2268 & -61 \\
\hline $4 / 17 / 20$ & 2224 & 1896 & 328 & 1070 & 1154 & 6230 & -4006 & 5825 & -3601 & 2456 & -232 & 3377 & -1153 & 2266 & -42 \\
\hline $4 / 18 / 20$ & 2235 & 1977 & 258 & 1117 & 1118 & 6816 & -4581 & 6345 & -4110 & 2510 & -275 & 3547 & -1312 & 2255 & -20 \\
\hline \multirow[t]{2}{*}{$4 / 19 / 20$} & 2235 & 2061 & 174 & 1165 & 1071 & 7458 & -5223 & 6910 & -4675 & 2563 & -328 & 3722 & -1487 & 2236 & -1 \\
\hline & & \multicolumn{2}{|c|}{$\mathrm{Q}_{1}$} & \multicolumn{2}{|c|}{$\mathrm{Q}_{2}$} & \multicolumn{2}{|c|}{$\mathrm{Q}_{3}$} & \multicolumn{2}{|c|}{$\mathrm{Q}_{4}$} & \multicolumn{2}{|c|}{$\mathrm{Q}_{5}$} & \multicolumn{2}{|c|}{$\mathrm{Q}_{1: 5}$} & \multicolumn{2}{|c|}{ GM } \\
\hline \multicolumn{2}{|c|}{$\mathrm{ME}^{(\mathrm{c})}$} & \multicolumn{2}{|c|}{471} & \multicolumn{2}{|c|}{1201.3} & \multicolumn{2}{|c|}{-2807.7} & \multicolumn{2}{|c|}{-2532.5} & \multicolumn{2}{|c|}{-138.82} & \multicolumn{2}{|c|}{-778.73} & \multicolumn{2}{|c|}{-60.8182} \\
\hline \multicolumn{2}{|c|}{$\operatorname{MAE}^{(\mathrm{d})}$} & \multicolumn{2}{|c|}{471} & \multicolumn{2}{|c|}{1201.3} & \multicolumn{2}{|c|}{2807.7} & \multicolumn{2}{|c|}{2532.5} & \multicolumn{2}{|c|}{138.82} & \multicolumn{2}{|c|}{778.73} & \multicolumn{2}{|c|}{60.8182} \\
\hline \multirow{2}{*}{\multicolumn{2}{|c|}{$\begin{array}{c}\operatorname{MAPE}^{(\mathrm{e})} \\
\operatorname{MSE}^{(\mathrm{f})}\end{array}$}} & \multicolumn{2}{|c|}{0.2229} & \multicolumn{2}{|c|}{0.5624} & \multicolumn{2}{|c|}{1.2894} & \multicolumn{2}{|c|}{1.1632} & & 632 & 0.3 & & 0.02 & \\
\hline & & 2.463 & $\times 10^{5}$ & 1.4467 & $\times 10^{6}$ & 9,61 & 6,653 & 7.7872 & $2 \times 10^{6}$ & & 503 & 7.6725 & $<10^{5}$ & 444 & \\
\hline
\end{tabular}

a. Estimations based on the best fitting $\left(y=0.126 \cdot x^{2.5809}, R^{2}=0.977\right)$, which is applied to the total time-series $\left(Q_{1: 5}\right)$ dataset. b. Gaussian model (GM: $\left.\left.y=2268 \cdot \exp \{-(x-51.28) / 22.72)^{2}\right\}, R^{2}=0.997\right)$, which is applied to the total time-series $\left(Q_{1: 5}\right)$ dataset. c. Mean error, $d$. Mean absolute error, e. Mean absolute percentage error, $f$. Mean square error. *. Cases shown in bold indicate better predictions of model $Q_{5}$ in comparison with the GM.

Table 2. Comparison between polynomial cubic and regression splines fitting curves with a complex network defined spline regression model-based the community detection proposed in this paper.

\begin{tabular}{ccccc}
\hline Model & $\mathbf{R}$ & $\mathbf{R}^{\mathbf{2}}$ & RMSE $^{(\mathbf{a})}$ & RAE $^{(\mathbf{b})}$ \\
\hline Cubic & 0.998 & 0.996 & 2.229 & $4.182 \%$ \\
Regression Splines with 3 random knots & 0.999 & 0.998 & 1.805 & $3.798 \%$ \\
Regression Splines with 4 random knots & 0.999 & 0.998 & 1.621 & $3.277 \%$ \\
Regression Splines with 5 random knots & 0.999 & 0.998 & 1.420 & $2.986 \%$ \\
Complex-Network Regression Splines (c) & 1.000 & 1.000 & 1.308 & $2.752 \%$ \\
\hline
\end{tabular}

a. Relative mean square error, b. Relative absolute error, c. Splines defined according to the community detection proposed in this paper. (source: [33]).

In a further study, the authors of [33] developed a spline regression model defined in the complex-network-based context proposed in this paper. In particular, the authors used the modularity communities configured in this paper to define the spline knot vector and to apply the spline regression algorithm to the cumulative Greek COVID-19 infection curve. The results are shown in Table 2, and they are compared with the cubic fitting of high determination and with regression splines of randomly selected $(3,4$ and 5$)$ knots.

As can be observed, the complex-network spline model built on modularity communities configured in this paper showed better determination ability and lower error terms than the cubic and the randomly calibrated splines models. These improvements in fitting ability were considerable even in the cases of $R$ and $R^{2}$, given the already good fitting performance of the cubic and the randomly calibrated spline models. These results verify the added value of the proposed in this paper methodological framework, which builds on conceptualizing a time-series as complex-network and separating it into densely intra-connected parts. In conceptual terms, these better results may be related with the immanent property of complex network analysis to model and manage problems of complexity and thus to provide better insights in the study complex systems, as in the case of the COVID-19 temporal spread.

\subsection{Policy Assessment}

At the final step, the communities resulting from the modularity optimization algorithm are examined in accordance with the anti-COVID-19 policies applied by the Greek state in the meantime. 
Table A1 (shown in the Appendix A) summarizes the evolution of the major policies of the state in the fight against the disease, which are grouped according to the communities resulting from the analysis. As can be observed, in the first three days $\left(Q_{1}\right)$ of evolution of the Greek COVID-19 infection curve, the state applied optional policy measures for the control of the disease. The first compulsory measure was taken at the end of the (intermediated) $Q_{2}$ period, applied at local scale, showing perhaps an alerted of the state for the changing dynamics of the disease. During the second half of the first $\left(Q_{1}\right)$ period, which is placed between the 9th to 19th days since the first infection, a set of compulsory measures was taken for the first time at the national scale, concerning suspension of educational, cultural, justice, and recreation functions and activities. At the next two periods $\left(Q_{3}\right.$ and $\left.Q_{4}\right)$ of exponential growth, more severe measures were applied. Measures of $Q_{3}$ period concern national suspension of gathering, religious, and tourism activities and of maritime transport. On the other hand, the defining measure of the $Q_{4}$ period was started at its beginning and concerns the national restriction of road transport. The defining measure of the final period $\left(Q_{5}\right)$ regards the control of transportation and particularly the national suspension of private air transport. Provided that the anti-COVID-19 policies are effective to the evolution of the pandemic with a 5-day (on average) up to the 14-day (maximum) time-lag, we construct Figure 6 to illustrate the ranges where policies included in each period $\left(Q_{1}, Q_{2}, Q_{3}, Q_{4}\right.$, and $\mathrm{Q}_{5}$ ) can be effective to the evolution of the infection curve.

As can be observed in Figure 6, all periods are effective within a range covering up to two successive periods. This implies that the visible (in this analysis) effect of the Greek anti-COVID-19 policies on the shape of the infection curve is due to the policies applied up to the fourth period (which include all of the anti-COVID-19 policies except the national suspension of private air transport, which was applied to the fifth period). Overall, the time evolution of the major Greek anti-COVID-19 policies signifies a timely alert of the Greek state to the cascading dynamics of the pandemic, in accordance with the structure of the time-periods of the Greek COVID-19 infection curve. The evolution in the change of type of the fitting curve, from power to polynomial, to power, to exponential, to a smoother exponential, and finally (up to the 43rd day of the disease spreading) to logarithmic reveals that anti-COVID-19 policies applied by the Greek state are currently succeeding in controlling the cascading spread of the disease. In terms of the Gaussian modeling of epidemics [8,9], this timely alert of the Greek state led to a faster decline of the infection curve, which is about to reach its peak and move into the second half of the bell-shaped distribution. Despite the modeling perspective, the complex-network analysis of the Greek COVID-19 infection curve suffices to provide insights for the structure of the pandemic, which cannot be detected macroscopically by fitting a single curve.

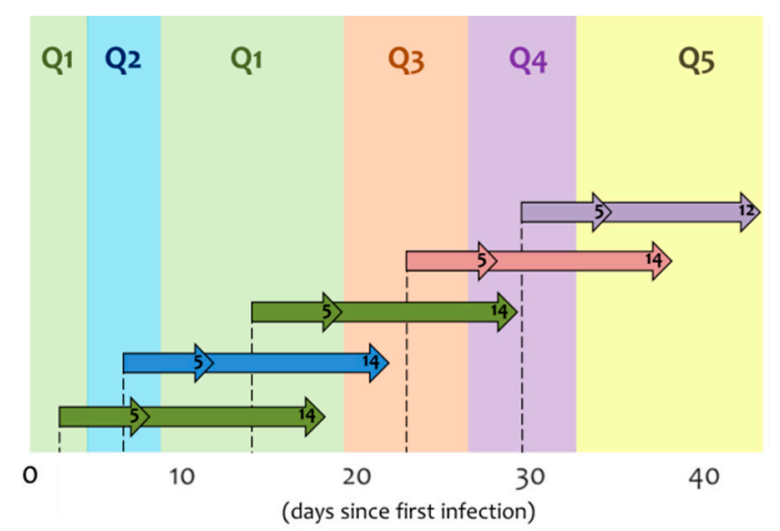

Figure 6. Lengths of the 5-day (on average) up to the 14-day (maximum) time time-lag expressing the time from exposition to the appearance of the symptoms of the disease. These lengths illustrate the ranges where policies included in each period $\left(Q_{1}, Q_{2}, Q_{3}, Q_{4}\right.$, and $\left.Q_{5}\right)$ can be effective to the evolution of the infection curve. 


\section{Conclusions}

The Greek anti-COVID-19 policies were applied just three days after the emergence of the first infected case in the country appear determinative to the evolution of the COVID-19 infection curve. The study built on complex network analysis of time-series (based on the visibility graph algorithm) to detect dynamics in the structure of the infection curve that are not visible by using classic time-series analysis methods, due to data limitation. Network analysis applied to the time series showed the existence of spatial constraints (expressed by development mainly of neighbor links) mixed with random trends, illustrating that (in the long-term) the evolution of the Greek COVID-19 infection curve resembles to a Markovian chain with slightly random transitions. In terms of forecasting, the structural analysis of the visibility graph detected stability of the hubs and instability of the medium and low-degree nodes, implying a low probability to meet maximum (infection) values at the future and high uncertainty in the variability of medium and lower values. Based on the modularity optimization algorithm, the Greek COVID-19 associated visibility graph is divided to five stages (periods) of different connectivity ruling the evolution of the pandemic in Greece. The first stage is described by a power pattern, the second (which intermediates the first one) is described by a second order polynomial (U-shaped) pattern, the third and fourth by exponential patterns, and a final one by a logarithmic pattern implying that the Greek COVID-19 infection curve tends to saturate. The resulting shape of the Greek COVID-19 infection curve to a logarithmic pattern can illustrate the effectiveness of the anti-COVID-19 policies applied in Greece, during the 43 days since the emergence of the first infected case. In terms of Gaussian modeling, this successive compression of the COVID-19 infection curve in five parts implies that the pandemic in Greece is about to reach the peak-time and move to the second (decline) half of the bell-shaped distribution. This measurable evolution of the COVID-19 infection curve makes it possible to consider Greece, at this early stage, a success story in the war against the pandemic. However, this good performance is temporary, and there is still a long way to go along the path of success. This is because, according to epidemiology, the case of pandemics only closes when the society develops either the appropriate herd immunity, which takes place through exposure to the disease, or clinical immunity, which takes place with the development of a vaccination. Within this context, there is still a long way to success, and in conjunction with the global expectations that the day of a cure to this pandemic is forthcoming, this paper provides insights into the current success story of Greece to inspire other countries towards more effective anti-COVID-19 policies and management.

Author Contributions: Conceptualization, D.T. and L.M.; Methodology, D.T. and L.M.; Software, D.T. and L.M.; Validation, D.T. and L.M.; Formal Analysis, D.T. and L.M.; Investigation, D.T. and L.M.; Resources, D.T. and L.M.; Data Curation, D.T. and L.M.; Writing-Original Draft Preparation, D.T.; Writing-Review \& Editing, D.T. and L.M.; Visualization, D.T.; Supervision, D.T. and L.M. All authors have read and agreed to the published version of the manuscript.

Funding: This research received no external funding.

Acknowledgments: The authors thank the Editor and the two anonymous reviewers for their valuable comments that improved the quality of this paper.

Conflicts of Interest: The authors declare no conflict of interest. 


\section{Appendix A}

Table A1. The evolution of major Greek anti-COVID-19 policies.

\begin{tabular}{|c|c|c|c|c|c|}
\hline Date & Community & n & $\begin{array}{c}\text { Daily } \\
\text { Infections }\end{array}$ & $\begin{array}{c}\text { Total } \\
\text { Infections }\end{array}$ & Policy Measure \\
\hline $2 / 27 / 20$ & \multirow{3}{*}{$\mathrm{Q}_{1}$} & 2 & 2 & 2 & \multirow{3}{*}{$\begin{array}{l}\text { Optional local suspension of schools operation at } \\
\text { regions with infected cases; Prohibition of school } \\
\text { excursions abroad, school decontaminations. }\end{array}$} \\
\hline $2 / 28 / 20$ & & 3 & 1 & 3 & \\
\hline $2 / 29 / 20$ & & 4 & 3 & 6 & \\
\hline $3 / 1 / 20$ & \multirow{4}{*}{$\mathrm{Q}_{2}$} & 5 & 2 & 8 & \multirow[b]{4}{*}{$\begin{array}{l}\text { Local suspension of schools, theaters, cinemas, and } \\
\text { cultural spaces at regions with infected cases. }\end{array}$} \\
\hline $3 / 2 / 20$ & & 6 & 0 & 8 & \\
\hline $3 / 3 / 20$ & & 7 & 0 & 8 & \\
\hline $3 / 4 / 20$ & & 8 & 2 & 10 & \\
\hline $3 / 5 / 20$ & \multirow{11}{*}{$\mathrm{Q}_{1}$} & 9 & 20 & 30 & \multirow{11}{*}{$\begin{array}{c}\text { National suspension of nursing houses, conferences, } \\
\text { and school excursions and indoors operation of } \\
\text { sport activities. } \\
\text { National suspension of cultural and art activities. } \\
\text { National suspension of schools and educational units. } \\
\text { National suspension of playgrounds, theaters, } \\
\text { cinemas, art and entertainment places. } \\
\text { National suspension of courts, malls, café, bars, } \\
\text { restaurants, museums, sport centers and } \\
\text { organized beaches. }\end{array}$} \\
\hline $3 / 6 / 20$ & & 10 & 15 & 45 & \\
\hline $3 / 7 / 20$ & & 11 & 20 & 65 & \\
\hline $3 / 8 / 20$ & & 12 & 7 & 72 & \\
\hline $3 / 9 / 20$ & & 13 & 11 & 83 & \\
\hline $3 / 10 / 20$ & & 14 & 5 & 88 & \\
\hline $3 / 11 / 20$ & & 15 & 10 & 98 & \\
\hline $3 / 12 / 20$ & & 16 & 18 & 116 & \\
\hline $3 / 13 / 20$ & & 17 & 73 & 189 & \\
\hline $3 / 14 / 20$ & & 18 & 38 & 227 & \\
\hline $3 / 15 / 20$ & & 19 & 103 & 330 & \\
\hline $3 / 16 / 20$ & \multirow{7}{*}{$\mathrm{Q}_{3}$} & 20 & 21 & 351 & \multirow{7}{*}{$\begin{array}{l}\text { National establishment of money penalty for } \\
\text { disobedience to the measures and of compulsory } \\
\text { 14-day quarantine to those entering the country. } \\
\text { National suspension of religion activities, closing of } \\
\text { national borders for tourism-related purposes. } \\
\text { National prohibition of public gathering ( }>10 \text { persons), } \\
\text { regulations to supermarket's entrance. } \\
\text { National suspension of hotels. } \\
\text { National restriction to maritime transport. }\end{array}$} \\
\hline $3 / 17 / 20$ & & 21 & 35 & 386 & \\
\hline $3 / 18 / 20$ & & 22 & 31 & 417 & \\
\hline $3 / 19 / 20$ & & 23 & 46 & 463 & \\
\hline $3 / 20 / 20$ & & 24 & 31 & 494 & \\
\hline $3 / 21 / 20$ & & 25 & 35 & 529 & \\
\hline $3 / 22 / 20$ & & 26 & 94 & 623 & \\
\hline $3 / 23 / 20$ & \multirow{6}{*}{$\mathrm{Q}_{4}$} & 27 & 71 & 694 & \multirow[t]{6}{*}{ National restriction of road transport. } \\
\hline $3 / 24 / 20$ & & 28 & 48 & 742 & \\
\hline $3 / 25 / 20$ & & 29 & 78 & 820 & \\
\hline $3 / 26 / 20$ & & 30 & 71 & 891 & \\
\hline $3 / 27 / 20$ & & 31 & 74 & 965 & \\
\hline $3 / 28 / 20$ & & 32 & 95 & 1060 & \\
\hline $3 / 29 / 20$ & \multirow{11}{*}{$\mathrm{Q}_{5}$} & 33 & 95 & 1155 & \multirow{11}{*}{ National suspension of private air transport. } \\
\hline $3 / 30 / 20$ & & 34 & 56 & 1211 & \\
\hline $3 / 31 / 20$ & & 35 & 102 & 1313 & \\
\hline $4 / 1 / 20$ & & 36 & 101 & 1414 & \\
\hline $4 / 2 / 20$ & & 37 & 129 & 1543 & \\
\hline $4 / 3 / 20$ & & 38 & 69 & 1612 & \\
\hline $4 / 4 / 20$ & & 39 & 60 & 1672 & \\
\hline $4 / 5 / 20$ & & 40 & 62 & 1734 & \\
\hline $4 / 6 / 20$ & & 41 & 20 & 1754 & \\
\hline $4 / 7 / 20$ & & 42 & 77 & 1831 & \\
\hline $4 / 8 / 20$ & & 43 & 52 & 1883 & \\
\hline
\end{tabular}

Source: Own elaboration based on government discourses and newspapers. 


\section{References}

1. Anderson, R.M.; Heesterbeek, H.; Klinkenberg, D.; Hollingsworth, T.D. How will country-based mitigation measures influence the course of the COVID-19 epidemic? Lancet 2020, 395, 931-934. [CrossRef]

2. McKibbin, W.J.; Fernando, R. The Global Macroeconomic Impacts of COVID-19: Seven Scenarios. CAMA Working Paper No. 19/2020. 2020. Available online: https://ssrn.com/abstract=3547729 (accessed on 22 June 2020).

3. World Health Organization. Coronavirus Disease 2019 (COVID-19); Situation Report 72; WHO: Geneva, Switzerland, 2020.

4. Wu, J.T.; Leung, K.; Bushman, M.; Kishore, N.; Niehus, R.; de Salazar, P.M.; Leung, G.M. Estimating clinical severity of COVID-19 from the transmission dynamics in Wuhan, China. Nat. Med. 2020, 26, 506-510. [CrossRef] [PubMed]

5. Li, M.; Chen, J.; Deng, Y. Scaling features in the spreading of COVID-19. arXiv 2020, arXiv:2002.09199.

6. Li, Y.; Liang, M.; Yin, X.; Liu, X.; Hao, M.; Hu, Z.; Jin, L. COVID-19 epidemic outside China: 34 founders and exponential growth. medRxiv 2020. [CrossRef]

7. Liu, Y.; Gayle, A.A.; Wilder-Smith, A.; Rocklöv, J. The reproductive number of COVID-19 is higher compared to SARS coronavirus. J. Travel Med. 2020, 27. [CrossRef]

8. Schlickeiser, R.; Schlickeiser, F. A Gaussian model for the time development of the sars-cov-2 corona pandemic disease. Predictions for Germany made on 30 March 2020. Physics 2020, 2, 164-170. [CrossRef]

9. Schuttler, J.; Schlickeiser, R.; Schlickeiser, F.; Kroger, M. Covid-19 predictions using a Gauss model, based on data from April 2. Physics 2020, 2, 197-212. [CrossRef]

10. Gao, J.; Tian, Z.; Yang, X. Breakthrough: Chloroquine phosphate has shown apparent efficacy in treatment of COVID-19 associated pneumonia in clinical studies. Biosci. Trends 2020, 14, 72-73. [CrossRef]

11. Gautret, P.; Lagier, J.C.; Parola, P.; Meddeb, L.; Mailhe, M.; Doudier, B.; Honoré, S. Hydroxychloroquine and azithromycin as a treatment of COVID-19: Results of an open-label non-randomized clinical trial. Int. J. Antimicrob. Agents 2020. [CrossRef]

12. Remuzzi, A.; Remuzzi, G. COVID-19 and Italy: What next? Lancet 2020, 395, 1225-1228. [CrossRef]

13. Roser, M.; Ritchie, H. Coronavirus Disease (COVID-19). Available online: https://ourworldindata.org/ coronavirus-data (accessed on 10 April 2020).

14. Livingston, E.; Bucher, K. Coronavirus disease 2019 (COVID-19) in Italy. JAMA 2020, 323, 1335. [CrossRef] [PubMed]

15. Watts, D.; Strogatz, D. Collective dynamics of small-world networks. Nature 1998, 393, 440-442. [CrossRef] [PubMed]

16. Christakis, N.A.; Fowler, J.H. Connected: The Surprising Power of our Social Networks and How They Shape Our Lives; Little, Brown and Company: New York, NY, USA, 2009.

17. Tsiotas, D. Detecting different topologies immanent in scalefree networks with the same degree distribution. Proc. Natl. Acad. Sci. USA 2019, 116, 6701-6706. [CrossRef] [PubMed]

18. Bedford, J.; Enria, D.; Giesecke, J.; Heymann, D.L.; Ihekweazu, C.; Kobinger, G.; Ungchusak, K. COVID-19: Towards controlling of a pandemic. Lancet 2020, 395, 1015-1018. [CrossRef]

19. Sohrabi, C.; Alsafi, Z.; O’Neill, N.; Khan, M.; Kerwan, A.; Al-Jabir, A.; Agha, R. World Health Organization declares global emergency: A review of the 2019 novel coronavirus (COVID-19). Int. J. Surg. 2020, 76, 71-76. [CrossRef]

20. Cohen, J.; Kupferschmidt, K. Countries test tactics in 'war'against COVID-19. Science 2020, 367, 1287-1288. [CrossRef]

21. Xu, B.; Gutierrez, B.; Mekaru, S.; Sewalk, K.; Goodwin, L.; Loskill, A.; Zarebski, A.E. Epidemiological data from the COVID-19 outbreak, real-time case information. Sci. Data 2020, 7, 1-6. [CrossRef]

22. Box, G.; Jenkins, G.M.; Reinsel, G.C.; Ljung, G.M. Time Series Analysis: Forecasting and Control; John Wiley \& Sons: Hoboken, NJ, USA, 2015.

23. Lacasa, L.; Luque, B.; Ballesteros, F.; Luque, J.; Nuno, J.C. From time-series to complex networks: The visibility graph. Proc. Natl. Acad. Sci. USA 2008, 105, 4972-4975. [CrossRef]

24. Tsiotas, D.; Charakopoulos, A. VisExpA: Visibility expansion algorithm in the topology of complex networks. SoftwareX 2020, 11, 100379. [CrossRef] 
25. Blondel, V.; Guillaume, J.-L.; Lambiotte, R.; Lefebvre, E. Fast unfolding of communities in large networks. J. Stat. Mech. 2008, 10, 10008. [CrossRef]

26. National Public Health Organization of Greece-NPHOG. New coronavirus Covid-19-Instructions. Available online: https://eody.gov.gr/neos-koronaios-covid-19 (accessed on 26 April 2020).

27. Ministry of Health of Greece-MOHG. Press Releases. Available online: https://www.moh.gov.gr/articles/ ministry/grafeio-typoy/press-releases (accessed on 30 April 2020).

28. Gao, Z.-K.; Small, M.; Kurths, J. Complex network analysis of time-series. Europhys. Lett. 2017, 116, 50001. [CrossRef]

29. Barabasi, A.L. Network Science; Cambridge University Press: Cambridge, UK, 2016.

30. Barthelemy, M. Spatial networks. Phys. Rep. 2011, 499, 1-101. [CrossRef]

31. Fortunato, S. Community detection in graphs. Phys. Rep. 2010, 486, 75-174. [CrossRef]

32. Walpole, R.E.; Myers, R.H.; Myers, S.L.; Ye, K. Probability \& Statistics for Engineers E Scientists; Prentice Hall Publications: New York, NY, USA, 2012.

33. Demertzis, K.; Tsiotas, D.; Magafas, L. Modeling and forecasting the COVID-19 temporal spread in Greece: An exploratory approach based on complex network defined splines. arXiv 2020, arXiv:2005.01163.

34. Fang, Y.; Zhang, H.; Xie, J.; Lin, M.; Ying, L.; Pang, P.; Ji, W. Sensitivity of chest CT for COVID-19: Comparison to RT-PCR. Radiology 2020, 200432. [CrossRef]

35. Heymann, D.L.; Shindo, N. COVID-19: What is next for public health? Lancet 2020, 395, 542-545. [CrossRef]

36. Chapra, S.C. Applied Numerical Methods with MATLAB for Engineers and Scientists; McGraw-Hill: New York, NY, USA, 2012.

37. Tsiotas, D.; Polyzos, S. The complexity in the study of spatial networks: An epistemological approach. Netw. Spat. Econ. 2018, 18, 1-32. [CrossRef]

(C) 2020 by the authors. Licensee MDPI, Basel, Switzerland. This article is an open access article distributed under the terms and conditions of the Creative Commons Attribution (CC BY) license (http://creativecommons.org/licenses/by/4.0/). 